\title{
Plasma acceleration and cooling by strong laser field due to the action of radiation reaction force
}

\author{
V. I. Berezhiani \\ Andronikashvili Institute of Physics, Tamarashvili 6, Tbilisi 0177, Georgia \\ S. M. Mahajan \\ Institute for Fusion Studies, The University of Texas at Austin, Austin, Texas 78712, USA \\ Z. Yoshida \\ Graduate School of Frontier Sciences, The University of Tokyo, Kashiwa 277-8561, Chiba, Japan
}

(Received 7 April 2008; published 11 December 2008)

\begin{abstract}
It is shown that for super intense laser pulses propagating in a hot plasma, the action of the radiation reaction force (appropriately incorporated into the equations of motion) causes strong bulk plasma motion with the kinetic energy raised even to relativistic values; the increase in bulk energy is accompanied by a corresponding cooling (intense cooling) of the plasma. The effects are demonstrated through explicit analytical calculations.
\end{abstract}

DOI: 10.1103/PhysRevE.78.066403

PACS number(s): 52.27.Ny, 52.27.Ep, 52.30.Ex, 52.38.Kd

\section{INTRODUCTION}

The bulk acceleration of a plasma to relativistic velocities represents one of the central issues of modern plasma physics and astrophysics. In most literature, devoted to the dynamics of plasmas embedded in the field of electromagnetic (EM) radiation, the radiation reaction force (RRF) acting on the plasma particles is neglected; it is ordered small compared to the Lorentz force, and is not expected to be a major determinant of plasma dynamics. There are two distinct cases when this assumption may not be justified: (1) Several astrophysical situations in which the spatiotemporal scales of plasma motion are sufficiently large, and (2) when the intensity of radiation is relativistically strong.

The modern petawatt lasers systems are already capable of producing ultrashort pulses with the focal intensities $I$ $=10^{21}-10^{22} \mathrm{~W} / \mathrm{cm}^{2}[1]$. Pulses of even higher intensities exceeding $I=10^{24} \mathrm{~W} / \mathrm{cm}^{2}[2]$ and lasting from a few femtoseconds to picoseconds (in the energy range $1 \mathrm{~J}-1 \mathrm{~kJ}$ ) are likely to be available soon. The power radiated by charged particles, accelerated in the EM field, increases with energy. Since the scattered radiation carries away the energy and momentum, the recoil force felt by the radiating charge (i.e., the radiation reaction, or the radiation "damping") could become significant. Recently Zhidkov et al. [3] studied the effect of radiation reaction on the interaction of an ultraintense laser pulse with an overdense plasma slab via a relativistic particle-in-cell simulation. Applying the Landau and Lifshitz expression for the force it was shown that the effects of radiation reaction become significant if the laser intensity exceeds $10^{22} \mathrm{~W} / \mathrm{cm}^{2}$ (corresponding to the normalized laser amplitude $e E_{0} / m_{e} c \omega \sim 50$, see also [4]). For higher intensities considerable modification of the scattered radiation spectrum is expected, in particular, a burst of incoherent $\mathrm{x}$ rays could be emitted.

The radiation pressure could also be important in astrophysical conditions. In fact, the acceleration of plasma by radiation pressure force has been considered as a possible mechanism for producing relativistic outflows (jets) from very luminous radiation sources, such as the active galactic nuclei (AGNs) or compact galactic objects (see [5] and ref- erences therein). In astrophysical community it is customary to use the term "the radiation pressure" as a pressure arising on the plasma particles through Compton scattering of incoherent photon fluxes generated by those objects. Through scattering of external photons, individual particles in a plasmas lose energy simultaneous with momentum transfer to the plasma. The bulk flow can be either accelerated or decelerated (i.e., radiative drag). The radiative drag force is derived by resorting to a phenomenological, test-particle approach. In this approach the energy-momentum conservation (in the Thompson or the Compton-Klein-Nishina regime) is invoked to treat the particle-photon interaction with subsequent integration of the obtained force over the distribution function.

It is interesting to remark that Landau and Lifshitz [6] (see also [7]) demonstrated that the radiation drag force, acting on an electron which scatters photons, can be derived (in the Thompson regime) not only through the energymomentum considerations but also by averaging the RRF. Thus, the test particle approach (based on semiclassical treatment of recoil arising from photon scattering) is equivalent to the classical treatment (including RRF in the equation of motion) as long as the photon energy in the particle rest frame $\hbar \omega \ll m c^{2}$, and the strength of EM fields is kept below the Schwinger limit. Though this statement sounds trivial, the discovery that the inclusion of radiation reaction in the equation of motion of a charged particle [in LorentzAbraham-Dirac (LAD) form [8], and variety of its approximations and/or modifications [6,9]] could, in certain cases, lead to particle energy gain (see, for instance, $[4,10,11]$ ) was labeled "surprising" and, in fact, counterintuitive. Indeed, the accelerated charged particles radiate the EM field, and consequently their motion should dampen. According to Zel'dovich [12] a systematic acceleration of charged particles, in the direction of EM wave propagation, is the main consequence of inclusion of RRF force in the equation of motion. The origin of this effect is related to the fact that the charged particle, absorbing energy from the wave, also absorbs a corresponding momentum. The energy is not accumulated by the particle but is reemitted. However, the reemitted energy is not "directed" exactly forward; the momentum lost by the particle is less than the momentum 
gain. Keitel et al. [13] conducted careful analytical and numerical analysis of the electron motion in the field of a strong laser pulse, and confirmed that the damping of electron motion takes place in the laser field polarization direction, while in the propagation direction electrons are accelerated due to the absorption and reabsorption of emitted radiation.

In this paper the radiative acceleration to relativistic velocities of a hot, optically thin, underdense plasma, exposed to a relativistic strong laser pulse, is investigated. We use the plasma dynamics model, recently developed in [14] in which the RRF is consistently included in the relativistic fluid equations. By taking moments of the relativistic kinetic equation (RRF in the Landau-Lifshitz form) the authors derived a manifestly covariant relativistic hydrodynamic equations for a multispecies relativistic plasma in an external EM field. It was found that the derived equations, in the limit of a plasma embedded in the field of incoherent photon fluxes, can be reduced to the equations for plasma bulk motion, obtained (in astrophysical context) in previous literature by adopting the test particle approach [5].

It has been shown by O'Dell [15] that a hot (relativistic) plasma, immersed in the photon fluxes from astrophysical objects, feels a much larger radiation pressure than a cold (nonrelativistic) gas, and tends to drive itself away from the radiation source with momentum derived largely from the anisotropic loss of its own internal energy. This "Compton rocket" is, however, always accompanied by "Compton cooling" of the plasma (see Phinney in Ref. [5]).

Similar effect could be operative in laser plasma as well. Laser sources generate highly coherent but anisotropic photon fluxes. We will soon demonstrate that ultrastrong laser pulses, impinging on a hot plasma, can indeed strongly accelerate as well as cool the bulk plasma via the action of RRF. It turns out that, under certain simplified assumptions, the effect can be demonstrated analytically.

\section{BASIC EQUATIONS}

We use the set of relativistic hydrodynamic equations derived in [14] for optically thin multispecies plasma. Throughout this paper, we adopt the notations and conventions used in [14] excepting the metric that is chosen to be $g^{\alpha \beta}$ $=(1,-1,-1,-1)$. The fluid equations, valid for each species, can be written in a manifestly covariant form

$$
\frac{\partial T^{\alpha \beta}}{\partial x^{\beta}}-q F^{\alpha \beta} n U_{\beta}=F_{\mathrm{rad}}^{\alpha},
$$

where the greek indices go from 0 to $3 ; \partial_{\alpha}=\partial / \partial x^{\alpha}$ $=\left(c^{-1} \partial / \partial t, \boldsymbol{\nabla}\right) ; T^{\alpha \beta}$ is the energy-momentum tensor of plasma species with charge $q$ and mass $M$, and $U^{\alpha}=(\gamma, \gamma \mathbf{V} / c)$ is the local four velocity with $\gamma=\left(1-V^{2} / c^{2}\right)^{-1 / 2}\left(U_{\alpha} U^{\alpha}=1\right)$; the rest-frame particle density $n$ satisfies the continuity equation $\partial n U^{\alpha} / \partial x^{\alpha}=0$. Note that we do not label the fluid species by an additional index for brevity. The EM field tensor can be formally written as $F^{\alpha \beta}=[\mathbf{E}, \mathbf{B}]$ and it satisfies the Maxwell equations $\partial_{\beta} F^{\alpha \beta}=-(4 \pi / c) J^{\alpha}, \quad \epsilon^{\alpha \beta \gamma \delta} \partial_{\beta} F_{\gamma \delta}=0$, where $J^{\alpha}$ $=(c \rho, \mathbf{J}), \rho$ and $\mathbf{J}$ are, respectively, the total charge and the current density of plasma and $\epsilon^{\alpha \beta \gamma \delta}$ is the antisymmetric tensor.

Equation (1), expressing the conservation of momentum and energy, has, in addition to the conventional terms (lefthand side), an extra term $F_{\text {rad; }}^{\alpha}$ the latter represents the 4-force density related to the radiation reaction (emission and/or scattering), and is necessary to correct the momentum energy balance. Note that the momentum change due to collisions is ignored in Eq. (1). This assumption can be justified by the fact that with increase of EM radiation intensity, the role of collisional processes decreases and they can be ignored in comparison to the radiation reaction that scales up with the intensity of the electromagnetic wave.

The energy momentum tensor $T^{\alpha \beta}$ is assumed to be that of an ideal isotropic fluid: $T^{\alpha \beta}=w U^{\alpha} U^{\beta}-g^{\alpha \beta} p$, where $w=E+p$ is the enthalpy per unit volume, and $E(p)$ is the proper internal energy density (pressure) of the fluid. For a Maxwellian plasma, $w=n c^{2} M G(\bar{z})$ while $p=n T \quad[16]$ with $G$ $=K_{3}(\bar{z}) / K_{3}(\bar{z})$ where $K_{2}$ and $K_{3}$ are, respectively, the modified Bessel functions of the second and third order. The argument $\bar{z}=M c^{2} / T$, and $T$ is the temperature measured in the rest frame of a fluid element. The function $G(\bar{z})$ defines the "effective" temperature-dependent mass of the particles, and has the following limiting expressions: $G \approx 1+5 / 2 \bar{z}$ for $\bar{z}$ $\gg 1$, and $G \approx 4 / \bar{z}$ for $\bar{z} \ll 1$. The expression for $F_{\text {rad }}^{\alpha}$ has been derived in [14], and is

$$
\begin{aligned}
F_{\mathrm{rad}}^{\alpha}= & \frac{2 q^{3}}{3 M^{2} c^{4}} \frac{\partial F^{\alpha \beta}}{\partial x^{\gamma}} T_{\beta}^{\gamma}+\sigma n\left\{[1+2 G(\bar{z}) / \bar{z}] \bar{T}^{\alpha \beta} U_{\beta}\right. \\
& \left.-[1+6 G(\bar{z}) / \bar{z}] \bar{T}^{\beta \gamma} U_{\beta} U_{\gamma} U^{\alpha}\right\},
\end{aligned}
$$

where $\sigma=8 \pi q^{4} / 3 M^{2} c^{4}$ is the Thomson cross section, and $\bar{T}^{\alpha \beta}=\frac{1}{4 \pi}\left(-F^{\alpha \gamma} F_{\gamma}^{\beta}+\frac{1}{4} g^{\alpha \beta} F^{\gamma \delta} F_{\gamma \delta}\right)$ is the energy momentum tensors of the EM field.

Equations (1) and (2) along with the continuity and Maxwell's equations form the closed set describing consistently the bulk motion of a multispecies relativistic plasma in the presence of strong EM radiation. It is convenient to introduce the following dimensionless variable: $x^{\prime \alpha}=(\omega / c) x^{\alpha}$ [i.e., $\left.\quad t^{\prime}=\omega t, \quad \mathbf{r}^{\prime}=(\omega / c) \mathbf{r}\right], \quad T^{\prime}=T / M c^{2} \quad\left(\bar{z}=1 / T^{\prime}\right), \quad F^{\prime \alpha \beta}$ $=(q / M c \omega) F^{\alpha \beta} \quad$ [i.e., $\left.\mathbf{E}^{\prime}=(q / M c \omega) \mathbf{E}, \mathbf{B}^{\prime}=(q / M c \omega) \mathbf{B}\right], n^{\prime}$ $=n / N_{0}, \quad p^{\prime}=p / N_{0} M c^{2}, \quad V^{\prime}=V / c, \quad$ and $\gamma=\sqrt{1-V^{\prime 2}}\left[U^{\prime a}\right.$ $\left.=\left(\gamma, \gamma \mathbf{V}^{\prime}\right)\right], \quad T^{\alpha \beta}=T^{\alpha \beta} /\left(N_{0} M c^{2}\right), \quad \bar{T}^{\prime \alpha \beta}=4 \pi(q / M c \omega)^{2} \bar{T}^{\alpha \beta}$, $J^{\prime \alpha}=J^{\alpha} /\left(q N_{0} c\right)$. In this notation, $N_{0}$ is the equilibrium density of the plasma and $\omega$ is the characteristic frequency of the EM field. Suppressing the superscripts, we arrive at the dimensionless equations

$$
\begin{gathered}
\frac{\partial T^{\alpha \beta}}{\partial x^{\beta}}-F^{\alpha \beta} n U_{\beta}=F_{\text {rad }}^{\alpha}, \\
F_{\mathrm{rad}}^{\alpha}=\varepsilon \frac{\partial F^{\alpha \beta}}{\partial x^{\gamma}} T_{\beta}^{\gamma}+\varepsilon n\left\{[1+2 G(\bar{z}) / \bar{z}] \bar{T}^{\alpha \beta} U_{\beta}\right. \\
\left.-[1+6 G(\bar{z}) / \bar{z}] \bar{T}^{\beta \gamma} U_{\beta} U_{\gamma} U^{\alpha}\right\},
\end{gathered}
$$

where $\varepsilon=\omega \tau_{0}$ and $\tau_{0}=2 q^{2} / 3 M c^{3}$ [for electrons $\left(M=m_{e}, q\right.$ $\left.=-|e|) \tau_{0}=0.6 \times 10^{-23} \mathrm{~s}\right]$. 
In order to explicitly display the content of these equations, it helps to go to the more familiar notation of space and time components. In what follows we work in terms of three vectors sacrificing manifest covariance. We begin by writing the EM field tensor in the matrix form

$$
\bar{T}^{\alpha \beta} \equiv\left[\begin{array}{cc}
W, & \mathbf{S} \\
\mathbf{S}, & -\sigma_{i k}
\end{array}\right],
$$

where $W=\bar{T}^{00}=\frac{1}{2}\left(\mathbf{E}^{2}+\mathbf{B}^{2}\right)$ is the EM field energy density, $\mathbf{S}$ $=[\mathbf{E} \times \mathbf{B}]$ is the pointing vector and $\sigma_{i k}$ is a stress tensor given by the expression $\sigma_{i k}=\left[-\frac{1}{2} \delta_{i k}\left(\mathbf{E}^{2}+\mathbf{B}^{2}\right)+E_{i} E_{k}+B_{i} B_{k}\right]$ $(i, k=1,2,3)$. Simple algebra leads to the following identity:

$$
\bar{T}^{\alpha \beta} U_{\beta}=\gamma\left[\begin{array}{c}
\left(\mathbf{E}^{2}+\mathbf{B}^{2}\right) / 2-\mathbf{V} \cdot(\mathbf{E} \times \mathbf{B}) \\
\mathbf{A}_{L 1}+\mathbf{V}\left(\mathbf{B}^{2}-\mathbf{E}^{2}\right) / 2
\end{array}\right],
$$

where $\mathbf{A}_{L 1}=(\mathbf{E} \times \mathbf{B})+\mathbf{B} \times(\mathbf{B} \times \mathbf{V})+\mathbf{E}(\mathbf{V} \cdot \mathbf{E})$. By similar manipulations, we derive $\bar{T}^{\beta \gamma} U_{\beta} U_{\gamma}=\gamma^{2} A_{L 2}+\frac{1}{2}\left(\mathbf{B}^{2}-\mathbf{E}^{2}\right)$, where $A_{L 2}=[\mathbf{E}+(\mathbf{V} \times \mathbf{B})]^{2}-(\mathbf{V} \cdot \mathbf{E})^{2}$, and

$$
U^{\alpha} \bar{T}^{\beta \gamma} U_{\beta} U_{\gamma}=\gamma^{2}\left(A_{L 2}+\frac{1}{2 \gamma^{2}}\left(\mathbf{B}^{2}-\mathbf{E}^{2}\right)\right)\left[\begin{array}{c}
\gamma \\
\gamma \mathbf{V}
\end{array}\right] .
$$

The remaining terms, appearing explicitly in Eqs. (3) and (4), are $T_{\beta}^{\gamma} \partial_{\gamma} F^{\alpha \beta}$ and $\partial_{\beta} T^{\alpha \beta}$. One can show that $T_{\beta}^{\gamma} \partial_{\gamma} F^{\alpha \beta}$ $=\gamma n G U_{\beta} d F^{\alpha \beta} / d t-p \partial_{\beta} F^{\alpha \beta}$ [here $\left.d / d t=\partial / \partial t+(\mathbf{V} \cdot \boldsymbol{\nabla})\right]$. Using the Maxwell equation $\partial_{\beta} F^{\alpha \beta}=-\Omega_{L}^{2} J^{\alpha} \quad$ [where $\Omega_{L}$ $=\left(4 \pi q^{2} N_{0} / M\right)^{1 / 2} / \omega$ is the normalized plasma frequency] and applying the useful relation $F^{\alpha \beta} U_{\beta}=\gamma(\mathbf{E} \cdot \mathbf{V}, \mathbf{E}+[\mathbf{V} \times \mathbf{B}])$, we get

$$
T_{\beta}^{\gamma} \frac{\partial F^{\alpha \beta}}{\partial x^{\gamma}}=\gamma^{2} n G\left[\begin{array}{c}
\mathbf{V} \frac{d \mathbf{E}}{d t} \\
\frac{d \mathbf{E}}{d t}+\left[\mathbf{V} \times \frac{d \mathbf{B}}{d t}\right]
\end{array}\right]+p \Omega_{L}^{2}\left[\begin{array}{l}
\rho \\
\mathbf{J}
\end{array}\right]
$$

while for $\partial_{\beta} T^{\alpha \beta}$ we have the identity

$$
\frac{\partial T^{\alpha \beta}}{\partial x^{\beta}}=n \gamma \frac{d}{d t}\left(G U^{\alpha}\right)-\frac{\partial p}{\partial x_{\alpha}} .
$$

The preceding preparation is enough to spell out Eq. (3) into space and time components; the spatial component reads as

$$
\frac{d}{d t}(G \gamma \mathbf{V})+\frac{1}{N} \boldsymbol{\nabla} p=\mathbf{E}+(\mathbf{V} \times \mathbf{B})+\mathbf{R},
$$

where

$$
\begin{aligned}
\mathbf{R}= & \varepsilon \frac{1}{\gamma \bar{z}} \Omega_{L}^{2} \mathbf{J}-\varepsilon \frac{2 G}{\bar{z}} \mathbf{V}\left(\mathbf{B}^{2}-\mathbf{E}^{2}\right)+\varepsilon G \gamma\left[\frac{d \mathbf{E}}{d t}+\left(\mathbf{V} \times \frac{d \mathbf{B}}{d t}\right)\right] \\
& +\varepsilon(1+2 G / \bar{z})[(\mathbf{E} \times \mathbf{B})+\mathbf{B} \times(\mathbf{B} \times \mathbf{V})+\mathbf{E}(\mathbf{V} \cdot \mathbf{E})] \\
& -\varepsilon \gamma^{2}(1+6 G / \bar{z}) \mathbf{V}\left\{[\mathbf{E}+(\mathbf{V} \times \mathbf{B})]^{2}-(\mathbf{V} \cdot \mathbf{E})^{2}\right\} .
\end{aligned}
$$

Here $N=\gamma n$ is the density in the laboratory frame and pressure is $p=N / \bar{z} \gamma(p=N T / \gamma$ in dimensional units). Equation (10) is the vector fluid equation of a relativistic plasma generalized to include the radiation reaction force $\mathbf{R}$.
The temporal (zero) component of Eq. (3) can be readily written, and we do not display it here. We will, instead, complete the fluid system by deriving the (equivalent) equation for entropy by projecting Eq. (3) "along" the four velocity $U_{\alpha}$. Multiplying (3) by $U_{\alpha}$, and using the obvious identity $F^{\alpha \beta} U_{\beta} U_{\alpha}=0$, we get

$$
U_{\alpha} \frac{\partial T^{\alpha \beta}}{\partial x^{\beta}}=U_{\alpha} F_{\mathrm{rad}}^{\alpha},
$$

that, in three vector notation, becomes ( $S$ is the entropy per particle)

$$
\begin{aligned}
\frac{d S}{d t}= & \varepsilon \Omega_{L}^{2}(\rho-\mathbf{V} \cdot \mathbf{J})-\varepsilon(2 G) \gamma^{-1}\left(\mathbf{B}^{2}-\mathbf{E}^{2}\right) \\
& -\varepsilon(4 G) \gamma\left\{[\mathbf{E}+(\mathbf{V} \times \mathbf{B})]^{2}-(\mathbf{V} \cdot \mathbf{E})^{2}\right\},
\end{aligned}
$$

where

$$
S=\ln \left[\gamma K_{2} \exp (\bar{z} G) / \bar{z} N\right]+\text { const. }
$$

Deriving Eq. (13) we used the relations $U_{\alpha} J^{\alpha}=\gamma(\rho-\mathbf{V} \cdot \mathbf{J})$ and identity [17]

$$
\frac{d}{d t} G-\frac{\gamma}{N} \frac{d}{d t}\left(\frac{N}{\bar{z} \gamma}\right)=\frac{1}{\bar{z}} \frac{d S}{d t} .
$$

One can see that without the RRF $(\varepsilon=0)$ the plasma dynamics is isentropic with a corresponding relativistic adiabatic equation of state. With radiation reaction, the entropy is no longer a constant along the streamline.

In dimensionless form the Maxwell equations are $\boldsymbol{\nabla} \times \mathbf{E}$ $=-\partial_{t} \mathbf{B}, \boldsymbol{\nabla} \cdot \mathbf{B}=\mathbf{0}$ and

$$
\begin{gathered}
\boldsymbol{\nabla} \cdot \mathbf{E}=-\Omega_{L}^{2} \rho, \\
\boldsymbol{\nabla} \times \mathbf{B}=\frac{\partial \mathbf{E}}{\partial t}-\Omega_{L}^{2} \mathbf{J} .
\end{gathered}
$$

These equations along with Eqs. (10) and (13), and the continuity equation

$$
\frac{\partial N}{\partial t}+\boldsymbol{\nabla} \cdot(N \mathbf{V})=0,
$$

represent the closed system of Maxwell and relativistic fluid equations.

The RRF force explicitly depends on the plasma temperature. It is interesting to remark that in the cold plasma limit $(T \rightarrow 0), \bar{z} \rightarrow \infty$, and $G \rightarrow 1$, the first two terms on the righthand side of Eq. (11) vanish and $\mathbf{R}$ reduces to the Landau and Lifshitz [6] expression for the force acting on a single particle:

$$
\begin{aligned}
\mathbf{R}= & \varepsilon \gamma\left[\frac{d \mathbf{E}}{d t}+\left(\mathbf{V} \times \frac{d \mathbf{B}}{d t}\right)\right]+\varepsilon[(\mathbf{E} \times \mathbf{B})+\mathbf{B} \times(\mathbf{B} \times \mathbf{V}) \\
& +\mathbf{E}(\mathbf{V} \cdot \mathbf{E})]-\varepsilon \gamma^{2} \mathbf{V}\left\{[\mathbf{E}+(\mathbf{V} \times \mathbf{B})]^{2}-(\mathbf{V} \cdot \mathbf{E})^{2}\right\} .
\end{aligned}
$$

For high temperatures, however, the Landau-Lifshitz expression is modified by the temperature-dependent factors, and new terms appear (11).

For ultrarelativistic temperature $T \gg 1(\bar{z} \rightarrow 0)$ the effective mass of the fluid element enhanced to $G \simeq 4 T$. Consequently 
the Lorentz force on a fluid element becomes weaker in contradistinction to the RRF at ultrarelativistic temperature,

$$
\begin{aligned}
\mathbf{R}= & \varepsilon \gamma^{-1} T \Omega_{L}^{2} \mathbf{J}+4 \varepsilon T \gamma\left[\frac{d \mathbf{E}}{d t}+\left(\mathbf{V} \times \frac{d \mathbf{B}}{d t}\right)\right] \\
& -8 \varepsilon T^{2} \mathbf{V}\left(\mathbf{B}^{2}-\mathbf{E}^{2}\right)+8 \varepsilon T^{2}[(\mathbf{E} \times \mathbf{B})+\mathbf{B} \times(\mathbf{B} \times \mathbf{V}) \\
& +\mathbf{E}(\mathbf{V} \cdot \mathbf{E})]-24 \varepsilon \gamma^{2} T^{2} \mathbf{V}\left\{[\mathbf{E}+(\mathbf{V} \times \mathbf{B})]^{2}-(\mathbf{V} \cdot \mathbf{E})^{2}\right\}
\end{aligned}
$$

that becomes stronger because it scales as $\sim T^{2}$ [see last three terms on the right-hand side of Eq. (20)]. At sufficiently high temperatures $T(\gg 1)$, RRF can match or even beat the Lorentz force.

The temperature enhancement of the radiation drag is consistent with the results of O'Dell [15] (see also [5]), who demonstrated that a hot (relativistic) plasma embedded in the photon fluxes from the astrophysical objects feels a much larger radiation pressure than a cold (nonrelativistic) gas. The main equations derived in Refs. [15,5] by test particle approach can be recovered by averaging Eqs. (3) and (4) over the random phases of incoherent "photons" [14]. In the averaging procedure the Lorentz force vanishes, and the high frequency components in $U^{\alpha}$ are ignored. Strictly speaking in such an averaging procedure, one neglects the effects related to the high-frequency pressure (ponderomotive pressure) arising, for instance, by averaging the magnetic part of the Lorentz force $[\sim(\mathbf{V} \times \mathbf{B})]$. However, the corresponding force is a gradient force which vanishes for the uniform spatiotemporal structure of the radiation fluxes, i.e., the case that is mostly considered in astrophysical situations.

For a monochromatic EM pulse, the dynamics of plasma acceleration is ruled by the Lorentz force, while in the expression of RRF all terms can be equally important. In most cases of interest RRF can be ignored since it is considerably smaller than the Lorentz force. Indeed, Eq. (20) contains a small parameter $\varepsilon=\omega \tau_{0}$ which (for electrons) can be estimated to be $\varepsilon=1.2 \times 10^{-8} / \lambda[\mu \mathrm{m}]$, where $\lambda[\mu \mathrm{m}]$ is the vacuum wavelength in $\mu \mathrm{m}$. Thus, for available laser sources $\varepsilon \simeq 10^{-8}-10^{-9}$ and consequently the action of RRF can be ignored even for the laser pulses with intensities as high as $I=10^{20} \mathrm{~W} / \mathrm{cm}^{2}$ (note that such intensities have already been achieved in several laboratories worldwide). It is only at higher intensities that the RRF becomes comparable to the Lorentz force. A feature RRF worth emphasizing is that it is a nonpotential force and the plasma particles will be left with residual energies after interaction with the entire pulse. In this case, the bulk plasma is set in motion. If the plasma is relativistically hot (or is heated by the laser pulse itself) the RRF is stronger and it could provide a mechanism for the plasma bulk energy to grow at the expense of its internal energy and intensive plasma cooling will be the consequence.

To extract all the above-mentioned physics, one will have to resort to numerical methods to solve the complex system of Eqs. (10)-(19). However, under certain simplified assumption, the system is amenable to analytical solutions.

\section{TRANSPARENT PLASMAS}

The simplest nontrivial example is the EM pulse propagation in an underdense unmagnetized electron-positron plasma. We have chosen this system not only for its relative simplicity, but also due to the fact that in the ultrarelativistic limit (due to the strong laser field or due to the relativistic temperatures), the electron-ion plasma also behaves similarly to the electron-positron one. The electron-positron plasma is further relevant because the electron-ion collisions give rise to copious pair creation via Bremsstrahlung photons, or by the trident processes [18].

We consider a transversely polarized 1D pulse $\mathbf{E}$ $=\left(\mathbf{E}_{\perp}, 0\right)$ propagating along $z$ axis in a highly transparent plasma $\left(\Omega_{L} \ll 1\right)$. The group velocity of such a pulse is $v_{g}$ $=\left(1-1 / \Omega_{L}^{2}\right)^{1 / 2} \simeq 1$. We assume that the EM pulse profile is "given" and does not change in the course of propagation. The equilibrium state of the plasma is characterized by an overall charge neutrality $N_{e 0}=N_{p 0}=N_{0}$, where $N_{e 0}$ and $N_{p 0}$ are the unperturbed number densities of the electrons and positrons. In most mechanisms for creating $e-p$ plasmas, the pairs appear simultaneously and due to the symmetry of the problem it is natural to assume that $T_{e 0}=T_{p 0}=T_{0}$, where $T_{e 0}$ and $T_{p 0}$ are the respective equilibrium temperatures. The longitudinal motion of plasma is driven by the ponderomotive pressure, and RRF. Being the same for the electrons and positrons, these forces do not cause charge separation. Because of the symmetry between the electron and positron fluids, their temperatures, being initially equal, will also remain equal during the evolution of the system. It follows, then, that $N_{e}=N_{p}=N$ and $T_{e}=T_{p}=T$, and consequently $E_{z}$ $=\rho=J_{z}=0$.

Assuming that all variables depend on $\tau=(t-z)$ and taking into account the relations $\mathbf{B}=\left[\hat{\mathbf{z}} \times \mathbf{E}_{\perp}\right], \mathbf{A}_{L 1}=\hat{\mathbf{z}} \mathbf{E}_{\perp}^{2}\left(1-V_{z}\right)$ and $A_{L 2}=\mathbf{E}_{\perp}^{2}\left(1-V_{z}\right)^{2}$ (where $\hat{\mathbf{z}}$ is the unit vector directed along $z$ axis), Eq. (10) can be reduced to the following equation for the transverse momentum of the fluid

$$
\begin{aligned}
\frac{d}{d \tau}\left(G \mathbf{P}_{\perp}\right)= & \mathbf{E}_{\perp}+\varepsilon G \frac{d \mathbf{E}_{\perp}}{d \tau}\left(\gamma-P_{z}\right) \\
& -\varepsilon(1+6 G / \bar{z}) \mathbf{E}_{\perp}^{2}\left(\gamma-P_{z}\right) \mathbf{P}_{\perp}
\end{aligned}
$$

Here $\mathbf{P}=\gamma \mathbf{V}$ is the momentum of the fluid. The evolution of the longitudinal component of the momentum is governed by

$$
\begin{aligned}
(\gamma- & \left.P_{z}\right) \frac{d}{d \tau}\left(G P_{z}\right)-\frac{\gamma}{N} \frac{d}{d \tau}\left(\frac{N}{\gamma \bar{z}}\right) \\
= & \mathbf{P}_{\perp} \cdot\left(\mathbf{E}_{\perp}+\varepsilon G \frac{d \mathbf{E}_{\perp}}{d \tau}\left(\gamma-P_{z}\right)\right)+\varepsilon \mathbf{E}_{\perp}^{2}\left(\gamma-P_{z}\right) \\
& \times\left[(1+2 G / \bar{z})-(1+6 G / \bar{z}) P_{z}\left(\gamma-P_{z}\right)\right]
\end{aligned}
$$

and of the entropy (13), by

$$
\frac{d S}{d \tau}=\bar{z}\left[\frac{d}{d \tau} G-\frac{\gamma}{N} \frac{d}{d \tau}\left(\frac{N}{\gamma \bar{z}}\right)\right]=-4 \varepsilon G \mathbf{E}_{\perp}^{2}\left(\gamma-P_{z}\right) .
$$

Note that in the system of equations (21)-(23) the charge and the mass of the particle species cannot be seen explicitly since they are hidden in normalizations employed in the first 
section. It is now convenient to attribute this system to the positron fluid so that the normalizations are with respect to $q=|e|$ and $M=m_{e}$. To write the corresponding set of electron equations, we need to make the trivial replacement in (21)-(23): $\mathbf{E}_{\perp} \Rightarrow-\mathbf{E}_{\perp}$ and $\mathbf{J}_{\perp} \Rightarrow-\mathbf{J}_{\perp}$. One can easily see that the longitudinal momentum of electrons $P_{z}^{e}=P_{z}$ while $\mathbf{P}_{\perp}^{e}=$ $-\mathbf{P}_{\perp}$ and consequently $\gamma^{e}=\gamma=\left(1+P_{z}^{2}+\mathbf{P}_{\perp}^{2}\right)^{1 / 2}$ and $V_{z}^{e}=V_{z}$.

Taking the scalar product of Eq. (21) with $\mathbf{P}_{\perp}$, subtracting the result from Eq. (22) and applying Eq. (23), we find

$$
\frac{d}{d \tau}\left(\frac{1}{G\left(\gamma-P_{z}\right)}\right)=\varepsilon \mathbf{E}_{\perp}^{2} G^{-2}(1+6 G / \bar{z}) .
$$

Equation (24) can be readily integrated. For the initial conditions (at $\tau=0$, the plasma is at rest), $\mathbf{P}_{\perp}=0=P_{z}$, while $T$ $=T_{0}\left[G=G_{0}\left(T_{0}\right)\right]$, the solution is

$$
G\left(\gamma-P_{z}\right)-K(\tau)=0,
$$

where

$$
K(\tau)=\frac{1}{\varepsilon \int_{0}^{\tau} \mathbf{E}_{\perp}^{2} G^{-2}(1+6 G / \bar{z}) d \tau^{\prime}+G_{0}^{-1}} .
$$

After straightforward algebra, Eq. (25) yields the important relations

$$
\begin{gathered}
P_{z}=\frac{G^{2}\left(1+\mathbf{P}_{\perp}^{2}\right)-K^{2}}{2 G K}, \\
\gamma=\frac{G^{2}\left(1+\mathbf{P}_{\perp}^{2}\right)+K^{2}}{2 G K} .
\end{gathered}
$$

Now we can simplify Eqs. (21) and (23) further. Using these relations and Eq. (25), the equation for the transverse momentum can be written as

$$
\frac{d}{d \tau}\left(\frac{G \mathbf{P}_{\perp}}{K}-\varepsilon \mathbf{E}_{\perp}\right)=\frac{\mathbf{E}_{\perp}}{K} .
$$

To find the equation for the evolution of the plasma temperature, we will invoke the entropy equation (23). Integrating the continuity equation, and assuming that $N(\tau=0)=1$ we obtain $N=\gamma /\left(\gamma-P_{z}\right)=\gamma G / K(\tau)$. Using this expression, and Eqs. (14) and (25), we find that the temperature evolves as

$$
\frac{d}{d \tau}[F(\bar{z}) K]=-4 \varepsilon \mathbf{E}_{\perp}^{2} K^{2} F(\bar{z}),
$$

where the function $F$ depends on temperature $T\left(=\bar{z}^{-1}\right)$ via

$$
F(\bar{z})=\frac{K_{2}(\bar{z}) \exp [\bar{z} G(\bar{z})]}{\bar{z} G(\bar{z})} .
$$

In what follows we integrate the basic system of ordinary differential equations (26)-(31). For simplicity the incident pulse will be taken to be circularly polarized; the electric field of the pulse is given by $\mathbf{E}_{\perp}=(\hat{\mathbf{x}} \sin \tau+\hat{\mathbf{y}} \cos \tau) E_{\perp}(\tau)$, where $E_{\perp}(\tau)$ is the slowly varying envelope $\left[\left(\partial_{\tau} E_{\perp}\right) / E_{\perp}\right.$ $\ll 1]$ and $\hat{\mathbf{x}}$ and $\hat{\mathbf{y}}$ are the unit vectors. Since, for this choice of the pulse, $\mathbf{E}_{\perp}^{2}=E_{\perp}^{2}(\tau)$ does not contain high harmonics, $K$ and $T$ are also slowly varying. Equation (29), then, yields

$$
\left(G \mathbf{P}_{\perp}\right)^{2}=\left(1+\varepsilon^{2} K^{2}\right) E_{\perp}^{2}(\tau) .
$$

Interestingly one can use the preceding equation to eliminate the transverse momentum in Eqs. (27) and (28) to obtain

$$
\begin{gathered}
P_{z}=\frac{G^{2}-K^{2}+\left(1+\varepsilon^{2} K^{2}\right) E_{\perp}^{2}(\tau)}{2 G K}, \\
\gamma=\frac{G^{2}+K^{2}+\left(1+\varepsilon^{2} K^{2}\right) E_{\perp}^{2}(\tau)}{2 G K}
\end{gathered}
$$

giving $P_{z}$ and $\gamma$ entirely in terms of the field intensity of the EM wave.

In the cold plasma limit $T \rightarrow 0\left(G=G_{0}=1\right)$, Eq. (26) yields

$$
\begin{aligned}
K & =\frac{1}{\varepsilon \int_{0}^{\tau} E_{\perp}^{2}\left(\tau^{\prime}\right) d \tau^{\prime}+1} \\
& =\left(\begin{array}{cc}
\left(\varepsilon E_{0}^{2} \tau+1\right)^{-1} & \text { if } 0<\tau<\tau_{d} \\
\left(\varepsilon E_{0}^{2} \tau_{d}+1\right)^{-1}=\mathrm{const} & \text { if } \tau>\tau_{d}
\end{array}\right) .
\end{aligned}
$$

Since $\varepsilon \ll 1$ and $K \leqslant 1$, we can neglect terms $\varepsilon^{2} K^{2}$ in (33) and (34). The plasma momentum in the body of the pulse (i.e., $\left.0<\tau<\tau_{d}\right)$ is found to be

$$
P_{z}=\frac{1+E_{0}^{2}-K^{2}}{2 K}=\frac{1}{2}\left(1+\varepsilon E_{0}^{2} \tau\right)\left(1+E_{0}^{2}\right)-\frac{1}{2\left(1+\varepsilon E_{0}^{2} \tau\right)}
$$

while the residual momentum acquired by the plasma after the pulse is gone is calculated to be

$$
P_{z}(\text { res })=\frac{1}{2}\left(1+\varepsilon E_{0}^{2} \tau_{d}\right)-\frac{1}{2\left(1+\varepsilon E_{0}^{2} \tau_{d}\right)} .
$$

Similarly,

$$
\gamma=\frac{1}{2}\left(1+\varepsilon E_{0}^{2} \tau\right)\left(1+E_{0}^{2}\right)+\frac{1}{2\left(1+\varepsilon E_{0}^{2} \tau\right)} \quad \text { if } 0<\tau<\tau_{d}
$$

and

$$
\gamma(\text { res })=\frac{1}{2}\left(1+\varepsilon E_{0}^{2} \tau_{d}\right)+\frac{1}{2\left(1+\varepsilon E_{0}^{2} \tau_{d}\right)} \quad \text { if } \tau>\tau_{d} .
$$

The expression for the plasma density variation $(\delta N=N$ $-1)$ is

$$
\delta N=\frac{\gamma}{K(\tau)}-1=\frac{1}{2}\left(1+\varepsilon E_{0}^{2} \tau\right)^{2}\left(1+E_{0}^{2}\right)-\frac{1}{2} \quad \text { if } 0<\tau<\tau_{d}
$$

and

$$
\delta N(\text { res })=\frac{1}{2}\left(1+\varepsilon E_{0}^{2} \tau_{d}\right)^{2}-\frac{1}{2} \quad \text { if } \tau>\tau_{d} .
$$

In the absence of RRF we recover the well-known results [19]. For instance, if $\varepsilon=0$, we have $P_{z}=E_{0}^{2} / 2$, and $P_{z}$ (res) $=0$, which implies that the plasma particles are temporally accelerated only inside the region occupied by the pulse; the result is a consequence of the fact that the magnetic part of the Lorentz force (that is responsible for the longitudinal 
acceleration of the plasma) is a potential force. The action of RRF manifest itself not only by the enhancement of acceleration but also as the residual momentum that the whole plasma acquires. The pulse leaves in its wake an accelerated plasma which follows the pulse; its momentum, energy, and density are given by Eqs. (37), (39), and (41).

The strength of the acceleration caused by RRF is determined by the parameter $\varepsilon E_{0}^{2}$ and the duration of the pulse. The generated plasma flow becomes relativistic $\left[P_{z}(\right.$ res $)$ $>1$ ] when the pulse duration $\tau_{d}>\tau_{\text {acc }}$, where the "acceleration" time is given by

$$
\tau_{\mathrm{acc}}=\frac{1}{\varepsilon E_{0}^{2}} .
$$

It turns out that the magnitude of the zero temperature results are quite insensitive to small but finite temperature $(T \ll 1)$. However, a new qualitative feature emerges for finite temperature plasmas; the acceleration, now, is accompanied by a cooling of the plasma. Indeed, in the nonrelativistic limit $\bar{z} \gg 1(T \ll 1)$ we can use the asymptotic form of the modified Bessel function $K_{2}(\bar{z}) \simeq(\pi / 2 \bar{z}) e^{-\bar{z}}$ and $G \simeq 1$ $+5 /(2 \bar{z})$ in Eq. (31), and approximate $F \simeq(\pi / 2)^{1 / 2} T^{3 / 2}$. On substituting $F$ into Eq. (30), and applying the relation $d\left(K^{-1}\right) / d \tau=\varepsilon E_{\perp}^{2}$, we get for the plasma temperature

$$
\begin{gathered}
T=\frac{T_{0}}{\left(\varepsilon E_{0}^{2} \tau+1\right)^{2}} \quad \text { if } 0<\tau<\tau_{d} \\
T_{d}=\frac{T_{0}}{\left(\varepsilon E_{0}^{2} \tau_{d}+1\right)^{2}} \quad \text { if } \tau>\tau_{d}
\end{gathered}
$$

implying that the cooling begins immediately with the pulse interaction and the temperature keeps its minimum value $\left(T_{d}\right)$ after the pulse is gone.

It should be emphasized that in the relatively simple scenario of the "given" pulse, cooling is inhomogeneous in the sense that it is limited to the region that the pulse occupies. In a self-consistent treatment (i.e., when the pulse undergoes structural changes and/or modification, or depletion), however, plasma cooling will be more profoundly inhomogeneous.

When the plasma has ultrarelativistic temperatures ( $T$ $\gg 1$ ), the temperature function $F \simeq 27 T^{2}$, and Eq. (26) simplifies to

$$
K=\frac{4 T_{0}}{6 \varepsilon T_{0} \int_{0}^{\tau} E_{\perp}^{2}\left(\tau^{\prime}\right) d \tau^{\prime}+1},
$$

while the equation for temperature (30) reduces to

$$
\frac{d}{d \tau}\left[T^{2} K(\tau)\right]=-4 \varepsilon E_{\perp}^{2} K^{2} T^{2}
$$

the latter can be readily integrated [using the relation $\left.d\left(K^{-1}\right) / d \tau=3 \varepsilon E_{\perp}^{2} / 2\right]$ for the temperature $T$,

$$
T=\frac{T_{0}}{\left(6 \varepsilon E_{0}^{2} T_{0} \tau+1\right)^{5 / 6}} \quad \text { if } 0<\tau<\tau_{d},
$$

$$
T_{d}=\frac{T_{0}}{\left(6 \varepsilon E_{0}^{2} T_{0} \tau_{d}+1\right)^{5 / 6}} \quad \text { if } \tau>\tau_{d} .
$$

It follows from (47) and (48) that even in this ultrahot limit the plasma temperature begins to fall via RRF as soon as the plasma begins to interact with the laser pulse; the temperature remains at the lowest value achieved after the pulse is long gone.

In this ultrarelativistic limit, Eq. (34) for $\gamma$ factor can be approximated by $\left(\varepsilon^{2} K^{2} \ll 1\right)$

$$
\gamma=\frac{(4 T)^{2}+K^{2}+E_{0}^{2}(\tau)}{2(4 T) K}
$$

Note that at the initial stage of interaction (small $\tau$ ), although $K \sim 4 T_{0} \gg 1$ is large, the inequality $\left(\varepsilon^{2} K^{2} \ll 1\right)$ still holds and cannot be violated for any known physical conditions because $\varepsilon \simeq 10^{-8}-10^{-9}$ is so exceedingly small for laser pulses.

From Eqs. (45)-(49) we can extract detailed expressions for the relativistic factor,

$$
\begin{aligned}
\gamma= & \frac{1}{2}\left(6 \varepsilon E_{0}^{2} T_{0} \tau+1\right)^{1 / 6}+\frac{1}{2\left(6 \varepsilon E_{0}^{2} T_{0} \tau+1\right)^{1 / 6}} \\
& +\left(6 \varepsilon E_{0}^{2} T_{0} \tau+1\right)^{11 / 6} \frac{E_{0}^{2}}{32 T_{0}^{2}}
\end{aligned}
$$

if $0<\tau<\tau_{d}$, and

$$
\gamma(\mathrm{res})=\frac{1}{2}\left(6 \varepsilon E_{0}^{2} T_{0} \tau_{d}+1\right)^{1 / 6}+\frac{1}{2\left(6 \varepsilon E_{0}^{2} T_{0} \tau_{d}+1\right)^{1 / 6}}
$$

if $\tau>\tau_{d}$. Evidently the high temperature not only enhances the bulk plasma acceleration, it also makes it faster; the time of acceleration $\tau_{\mathrm{acc}}=1 /\left(6 \varepsilon E_{0}^{2} T_{0}\right)$ is reduced by the factor $T_{0}(\gg 1)$. We would like to reiterate that the acceleration of the plasma is augmented by its intensive cooling (47) and (48). The physical origin of the bulk motion can be traced to the fact that the hot particles in the fluid cell radiate most of their energy in the direction of the radiation source, and part of the energy of relativistic thermal motion is converted into the bulk motion. One must beware that cooling reduces the temperature, and at some stage, the relativistic temperature approximation will be violated especially if the duration of the pulse far exceeds the acceleration time $\tau_{d} \gg 6 \varepsilon E_{0}^{2} T_{0}$. For long pulses, therefore, we must numerically solve the problem to obtain quantitative results. Qualitative features of the system are not likely to be much affected since we have already shown that the acceleration goes on (but at a slower ate) even for temperatures that are nonrelativistic.

Let us now go back to the cold plasma and estimate the acceleration and/or cooling efficiency that depends on the following dimensionless parameters $\varepsilon, E_{0}, T_{0}$, and $\tau$. The strength of the radiation field is related to the intensity by $I$ $=(c / 4 \pi)\left\langle E_{\perp}^{2}\right\rangle$ were $\langle\cdots\rangle$ means averaging over the radiation high frequency periods. In terms of intensity, the dimensionless strength of the field $E_{0}\left(=e E_{0} / m c \omega\right.$ in units $)$ is given by 


$$
E_{0}^{2}=3.65 \times 10^{-19} I\left[\mathrm{~W} / \mathrm{cm}^{2}\right] \times \lambda^{2}[\mu \mathrm{m}]
$$

implying that the acceleration time (42), the time required for plasma flow to acquire relativistic velocities, can be estimated as

$$
\tau_{\mathrm{acc}}[\mathrm{fs}]=\frac{1.2 \times 10^{26}}{I\left[\mathrm{~W} / \mathrm{cm}^{2}\right]}=\frac{1.2 \times 10^{5}}{I_{21}},
$$

where $I_{21}=I\left[\mathrm{~W} / \mathrm{cm}^{2}\right] / 10^{21}$. Note that $\tau_{\text {acc }}$ does not depend on the frequency of the EM field. At currently achievable intensities $I=10^{21} \mathrm{~W} / \mathrm{cm}^{2}, E_{0} \approx 20$ for $\lambda=1 \mu \mathrm{m}$ leading to a $\tau_{\text {acc }}=100$ ps. Since $\tau_{\text {acc }}$ is much longer than the typical durations of such laser pulses, radiative acceleration will be minimal and not quite observable. However, for the future superintensity pulses, $\tau_{\text {acc }}$ could be considerably smaller than $\tau_{d}$. Indeed, for the laser field intensity $I=10^{23} \mathrm{~W} / \mathrm{cm}^{2}\left(E_{0}\right.$ $\approx 190$ for $\lambda=1 \mu \mathrm{m})$ we have $\tau_{\text {acc }} \sim 1 \mathrm{ps}$, while for $I$ $=10^{24} \mathrm{~W} / \mathrm{cm}^{2}\left(E_{0} \approx 600\right), \tau_{\text {acc }}$ will be several tens of femtoseconds. Then, plasma will be strongly accelerated acquiring relativistic bulk motion whose magnitude may be estimated to be $\gamma_{\mathrm{res}}=\tau_{d} / \tau_{\mathrm{acc}} \gg 1$.

Here we would like to emphasize that the validity of the classical treatment of the problem must be checked carefully for super-intense fields. Classical treatment of the problem is valid provided the energy of the photon involved in the process of interaction is smaller than the electron energy, i.e., $L=\hbar \omega_{e m} / \gamma_{e} m_{e} c^{2}<1$ (for ultrarelativistic case $\gamma_{e}=E_{0}$ ). Since the characteristic frequency of the photons emitted by accelerated electrons in the field of a circularly polarized laser field is $\omega_{e m} \simeq E_{0}^{3} \omega$, the factor $L \simeq 0.1$ is for $I=10^{23} \mathrm{~W} / \mathrm{cm}^{2}$ approaching $L \simeq 1$ when the intensities rise to $I$ $=10^{24} \mathrm{~W} / \mathrm{cm}^{2}$. Thus, for $I \geqslant 10^{24} \mathrm{~W} / \mathrm{cm}^{2}$, classical treatment becomes questionable. Recently Bulanov et al. (see Ref. [4]) demonstrated phenomenologically that inclusion of quantum effects in the RRF do not alter significantly the result obtained via the classical approximation up to laser intensities $I=1.38 \times 10^{25} \mathrm{~W} / \mathrm{cm}^{2}\left(E_{0}=2500\right.$ for $\left.\lambda=1 \mu \mathrm{m}\right)$. In general, rigorous treatment of quantum correction in the problem of $\mathrm{RRF}$ is challenging and is beyond the scope of the current paper.

Our entire calculation has been based on the "given field" approximation. This approximation, surely, breaks down when a large part of the radiation energy is converted into the kinetic energy of the plasma, or is scattered away. We will now derive the conditions under which the "given field" approach may be valid. Assuming that the radiation losses due to scattering are the same order as the energy needed for plasma acceleration, we can make a reasonable estimate of depletion length for the pulse. Comparing the initial energy of the pulse with the plasma residual energy,

$$
\frac{E_{\perp}^{2}}{4 \pi} c T_{L} \approx m_{e} c^{2} N \gamma_{\mathrm{res}} L_{D}
$$

we find for the pulse depletion length $L_{D}$,

$$
L_{D} \approx D\left(c \tau_{d}\right) \text {, }
$$

where

$$
D=\frac{\tau_{\mathrm{acc}}}{\tau_{d}} \frac{N_{c}}{N} E_{0}^{2} .
$$

Here $N_{c}$ is the critical density of the plasma. For strong acceleration we should have $\left(\tau_{\text {acc }} / \tau_{d}\right) \ll 1$ but for a transparent plasma, the critical density $N_{c} \gg N$. With $E_{0}^{2} \gg 1, D$ can be considerably larger unity implying that the pulse depletion length can be much longer than the pulse width. Thus, if the plasma slab is shorter than the $L_{D}$, the laser pump depletion may be ignored and the ignored, i.e., the "given field" approximation will give approximately correct results.

By dwelling on a relatively simple hot optically thin, underdense electron-positron plasma, exposed to a relativistic strong laser pulse, we have analytically demonstrated that the radiation reaction force (that becomes stronger with high tempearture) can cause an increase in the bulk kinetic energy of the fluid (that may be boosted up to relativistic values) accompanied by a corresponding cooling or intense cooling as the case may be.

When the super-intensity laser pulses $\left(I \geqslant 10^{24} \mathrm{~W} / \mathrm{cm}^{2}\right)$ become available in the future, it will be possible to create conditions in the laboratory such that the workings of the mechanism of the simultaneous gain of kinetic energy and loss of thermal energy (conjectured to play a fundamental role in advancing understanding of a variety of astrophysical phenomena) could be explored; the predictions of this and similar works can, then, be experimentally tested.

There are many ways by which this simple analytical work can be augmented. More detailed studies should be undertaken that include: (1) fluids with different masses, (2) denser plasmas, (3) giving up the "given field" approximation (a self-consistent calculation where the field intensity is also evolved), and (4) multidimensional effects.

We end this paper by giving the gist of our preliminary studies on an electron-ion plasma irradiated by super-strong laser pulses. We will continue assuming a radiation intensity of $\left(I \geqslant 10^{24} \mathrm{~W} / \mathrm{cm}^{2}\right)$. We find that the electrons are cooled and accelerated rapidly to relativistic speeds while ions lag behind owing to their larger inertia. The ensuing strong charge separation electrostatic fields cause a considerable slow down of the process of electron acceleration initially. However, at the subsequent stage, the strong charge separation field effectively accelerates the ions. When the ions catch up with the electrons the charge separation field becomes small, and the electrons are, once again, rapidly reaccelerated by the radiation field. The reported sequence of events takes place at $\tau_{\text {acc }}$.

\section{ACKNOWLEDGMENTS}

We thank Professor K. Mima for his interest in this problem and useful discussions. V.I.B. wishes to acknowledge the hospitality of the Graduate School of Frontier Sciences of the University of Tokyo, where most of the work was carried out. S.M.M. acknowledges support form USDOE Contract No. DE-FG02-04ER-54742. The work of V.I.B. was partially supported by Georgian NSF grant projects GNSF 69/07 (Grant No. GNSF/ST06/4-057) and GNSF 195/07 (Grant No. GNSF/ST07/4-191). 
[1] M. P. Perry et al., Opt. Lett. 24, 160 (1999); G. A. Mourou, C. P. J. Barty, and M. D. Perry, Phys. Today 51, 22 (1998); S. P. Hatchett et al., Phys. Plasmas 7, 2076 (2000).

[2] G. A. Mourou, T. Tajima, and S. V. Bulanov, Rev. Mod. Phys. 78, 309 (2006); M. Marklund and P. K. Shukla, ibid. 78, 591 (2006).

[3] A. Zhidkov, J. Koga, A. Sasaki, and M. Uesaka, Phys. Rev. Lett. 88, 185002 (2002).

[4] S. V. Bulanov, T. Zh. Esirkepov, J. Koga, and T. Tajima, Plasma Phys. Rep. 30, 196 (2004); J. Koga, T. Zh. Esirkepov, and S. V. Bulanov, Phys. Plasmas 12, 093106 (2005).

[5] M. Sikora and D. B. Wilson, Mon. Not. R. Astron. Soc. 197, 529 (1981); E. S. Phinney, ibid. 198, 1109 (1982); S. Inoue and F. Takahara, Prog. Theor. Phys. 98, 807 (1997); P. Madau and C. Thompson, Astrophys. J. 534, 239 (2000); L. Zampiery, R. Turolla, L. Foschini, and A. Treves, ibid. 592, 368 (2003).

[6] L. Landau and E. Lifshitz, The Classical Theory of Fields (Addison-Wesley, Reading, MA, 1959).

[7] J. J. Sanderson, Phys. Lett. 18, 114 (1965); T. W. B. Kibble, ibid. 20, 627 (1966); G. Schmidt, Phys. Lett. 74A, 222 (1979).

[8] F. Rohrlich, Clasical Charged Particles (Addison-Wesley, Reading, MA, 1965).

[9] F. Rohrlich, Phys. Lett. A 283, 276 (2001); H. Spohn, Europhys. Lett. 50, 287 (2000).

[10] F. V. Hartemann and A. K. Kerman, Phys. Rev. Lett. 76, 624
(1996); F. V. Hartemann, D. J. Gibson, and A. K. Kerman, Phys. Rev. E 72, 026502 (2005).

[11] J. E. Gunn and J. P. Ostriker, Astrophys. J. 165, 523 (1971); Ya. B. Zel'dovich and A. F. Illarionov, Zh. Eksp. Teor. Fiz. 61, 880 (1971) [Sov. Phys. JETP 34, 467 (1972)]; A. D. Steiger and C. H. Woods, Phys. Rev. D 5, 2912 (1972); D. M. Fradkin, Phys. Rev. Lett. 42, 1209 (1979).

[12] Ya. B. Zel'dovich, Usp. Fiz. Nauk 115, 161 (1975) [Sov. Phys. Usp. 18, 79 (1975)].

[13] C. H. Keitel, C. Szymanowski, P. K. Knight, and A. Maquet, J. Phys. B 31, 75 (1998).

[14] V. I. Berezhiani, R. D. Hazeltine, and S. M. Mahajan, Phys. Rev. E 69, 056406 (2004).

[15] S. L. O’Dell, Astrophys. J. 243, L147 (1981).

[16] V. I. Berezhiani and S. M. Mahajan, Phys. Rev. E 52, 1968 (1995); V. I. Berezhiani, S. M. Mahajan, Z. Yoshida, and M. Ohhashi, ibid. 65, 047402 (2002).

[17] J. Synge, The Relativistic Gas (North-Holland, Amsterdam, 1957).

[18] E. P. Liang, S. C. Wilks, and M. Tabak, Phys. Rev. Lett. 81, 4887 (1998); B. Shen and J. Meyer-ter-Vehn, Phys. Rev. E 65, 016405 (2001); V. I. Berezhiani, D. P. Garuchava, and P. K. Shukla, Phys. Lett. A 360, 624 (2007).

[19] N. L. Tsintsadze, Phys. Lett. 50, 33 (1974); M. AshourAbdalla, J. N. Leboeuf, T. Tajima, J. M. Dawson, and C. F. Kennel, Phys. Rev. A 23, 1906 (1981); V. I. Berezhiani, L. N. Tsintsadze, and P. K. Shukla, J. Plasma Phys. 48, 139 (1992). 